\title{
Threat Modeling Using Fuzzy Logic Paradigm
}

\author{
A. S. Sodiya, S. A. Onashoga, and B. A. Oladunjoye \\ Department of Computer Science, University of Agriculture, \\ Abeokuta, Nigeria
}

\section{sinaronke@yahoo.co.uk; bookyy2k@yahoo.com; oladunjoyeak@yahoo.com}

\begin{abstract}
Threat modeling, which is the process of identifying, quantifying and analyzing potential threats of computer-based systems, has become a significant consideration towards designing secure software systems. Despite the previous methods adopted for threat modeling, there are still many systems that are probable to attack. In this work, a fuzzy logic-based threat modeling technique is designed. The technique involves the fuzzification of input variables that is based on six major categories of threats (STRIDE- Spoofing, Tampering, Repudiation, Information Disclosure, Denial of Service, and Elevation of Privilege), rule evaluation, and aggregation of the rule outputs. The design is based on Mamdani-style inference system which is very good for the representation of human reasoning and effective analysis. The implementation is done using MATLAB fuzzy logic tools. Using the design to test five computer systems, the result shows a tool that can be effectively used to analyze potential threats to computer-based systems.
\end{abstract}

Keywords: Fuzzy logic, threat, threat modelling

\section{Introduction}

Nowadays, risk analysis plays a significant role in security management efforts. It was explained in Davis et al., 2004 that risk management is a tool that can help in production of secure software. In other words, security risk analysis is crucial to producing secure software products.

It is widely accepted that designing secure computer system is a difficult problem. Attackers frequently break into systems and cause a lot of destruction and as a result, software users are now interested in secure software products. In the past, many techniques have been developed to solve a wide array of security problems. Poorly chosen security measures can prove to be useless, or even counterproductive in the face of a well-executed attack. As such, the security system designers must be interested in incorporating security into every stage of software design.

Also, in order to adequately protect computer systems, it is important to properly understand the system and its associated potential threat. There are several processes for identifying and prioritizing risk. One of the most effective is threat modeling. Threat modeling is the process of identi-

Material published as part of this publication, either on-line or in print, is copyrighted by the Informing Science Institute. Permission to make digital or paper copy of part or all of these works for personal or classroom use is granted without fee provided that the copies are not made or distributed for profit or commercial advantage AND that copies 1) bear this notice in full and 2) give the full citation on the first page. It is permissible to abstract these works so long as credit is given. To copy in all other cases or to republish or to post on a server or to redistribute to lists requires specific permission and payment of a fee. Contact Publisher@InformingScience.org to request redistribution permission. fying, quantifying and analyzing potential threats of a computer-based system. It is a process of assessing and documenting a system's security risks (Ambler, 2005). It involves identifying the key assets of an application, decomposing the application, identifying and categorizing the threats to each assets or component, rating the threats based on a risk ranking, and then developing threat mitigation strategies that are then im- 
plemented in design, code and test cases (Davis et al., 2004).

Categorizing threats is the first step toward effectives mitigation (Ambler, 2005). Threats can be classified into six classes based on their effect (Swiderski et al., 2004):

1. Spoofing: - Using someone else credentials to gain access to otherwise inaccessible assets. A process mounts a spoofing attack by passing forged or stolen credentials.

2. Tampering: - Changing data to mount an attack.

3. Repudiation: - Occurs when a user denies performing an action, but the target of the action has no way to prove otherwise.

4. Information disclosure: - The disclosure of information to a user who does not have permission to see it.

5. Denial of service: - threatens the ability of valid users to access resources.

6. Elevation of privilege: - Occurs when an unprivileged user gain privileged status.

This is generally referred to as the STRIDE model. The STRIDE (Spoofing, Tampering, Repudiation, Information disclosure, Denial of Service, and Elevation of privilege) model was used by Microsoft for categorizing threats (Casteele, 2005). The model is used by developers and designers to identify and resolve security issues in the application code. This means that the STRIDE model is used to categorize the threats by taking into account their effects on the security of the application (Casteele, 2005).

The process of threat modeling helps system architects assess and document the security risks associated with a system. Identifying threats helps to develop efficient, realistic and meaningful security requirements for computer-based systems. This is particularly important because if the security requirements are faulty, then the system cannot be secure. Proper identification of threats and appropriate selection of countermeasures helps reduce the ability of attackers to misuse the system. Rigorous analysis of security requirements can detect security design flaws, allowing their correction prior to costly development and deployment of flawed systems. In this work, a fuzzy logic-based technique is designed for effective threat modeling.

The rest of this work is organized as follows. Section 2 present related works. In section 3, the methodology of the design is discussed. Section 4 presents the implementation and evaluation procedure. Future work and conclusion are presented in section 5.

\section{Literature Review}

Threat modeling is a sound approach to addressing software risks at the design level (Hoglund, 2004). Based on the decomposition of application, it evaluates the threats and risks to a system and chooses techniques to mitigate the threats. Security threats are modeled by attack trees, which describe the decision - making process attackers would go through to compromise the system.

Casteele (2005) presented a paper on threat modeling for web applications. This work focused on the important OWASP (Open Web Application Security Portal) Top 10 Web application security vulnerabilities/ problems. An architecture that is based on STRIDE model was used for the analysis. Klein (2005) presented a similar technique of carrying out threat modeling on a voting system.

Several academic teams jointly wrote a paper on analysis of threats that occurred when smart cards are used in web applications (De Cock et al., 2004). Their analysis was a part of the Designing Secure Applications (DeSecA) project, funded by Microsoft. The aim of their project was to provide an application developer with a tool that allows him to prevent the exploitation of a broad 
range of threats. They investigated common threats in five areas, each focusing on one particular technological building block for web applications. One of these was the smart card, and in particular the electronic identity card (De Cock et al., 2004).

Sodiya et al. (2006) presented an architecture for producing secure software and threat modeling was the foundation of this architecture. A comprehensive internet browser threat model was discussed in Griggs, 2004. He first listed various well known threat models on the internet and then identified the ones specific to browsers. The main types of attacks discussed are phishing, eavesdropping, man-in- the- middle, and denial of service (DoS).

Most of the papers discussed threat modeling for applications and how to incorporate it into development process of application products (quantitative). Our work differs from them in that it provides a systematic approach(qualitative) to threat modeling for computer-based systems using six categories of potential threats ( STRIDE model).

\section{Design Methodology}

The system is designed using fuzzy inference system which is a popular computing framework based on the concept of fuzzy set theory, fuzzy if - then rules, and fuzzy reasoning.

\section{The Model}

The fuzzy logic-based threat modeling architecture is given in Figure 1.

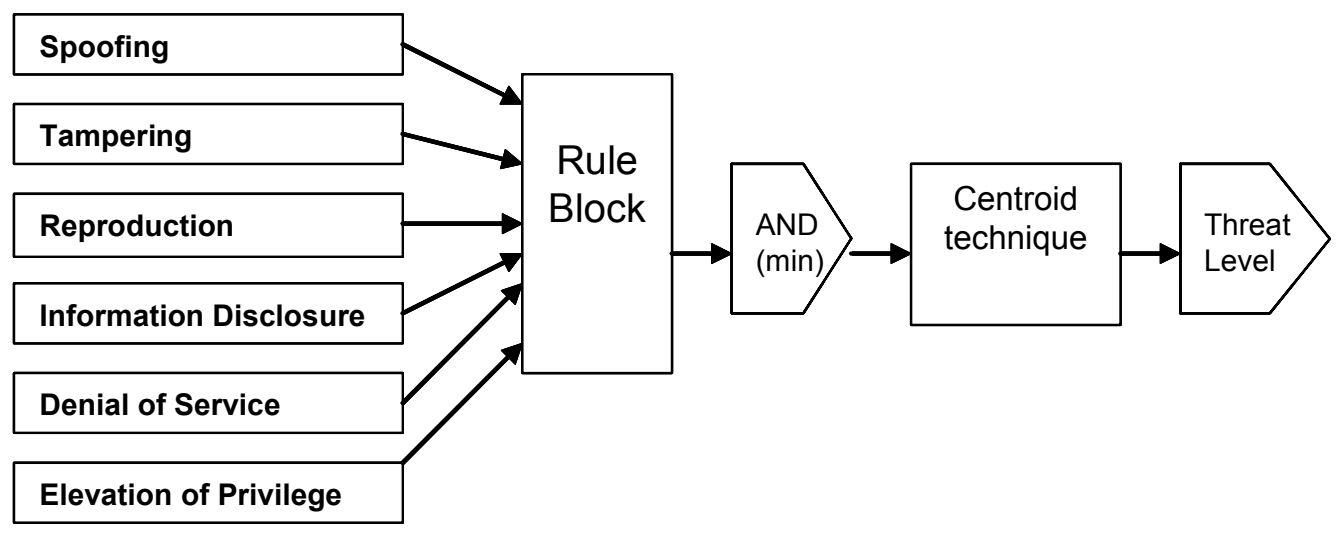

Figure 1: Architecture for Fuzzy Logic-based threat modelling

The steps involved in the design are:

\section{Fuzzification $\rightarrow$ Rule Evaluation $\rightarrow$ Aggregation $\rightarrow$ Defuzzification $\rightarrow$ Output}

a. Fuzzification: This is the process of generating membership values for a fuzzy variable using membership functions. The first step is to take the crisp inputs (Spoofing, Tampering, Repudiation, Information Disclosure, Denial of Service and Elevation of Privilege) and determine the degree to which these inputs belong to each appropriate fuzzy set. This crisp input is always a numeric value limited to the universe of discourse. Once the crisp inputs are obtained, they are fuzzified against the appropriate linguistic fuzzy sets.

Membership function is designed for each potential threat which is a curve that defines how each point in the input space is mapped to a membership value (or degree of membership) between 
$[0,1]$. Linguistic values are assigned for each threat as Low, Moderate, and High while for threat level as Very low, Low, Rather low, Medium Rather high, High, and Very high. For each input their values ranges from 0 to 10 while for output, ranges from 0 to 100 .

The following tables contain the linguistic variables and their ranges

\section{Linguistic variable: Spoofing, $S$}

\begin{tabular}{lc} 
Linguistic value & Numerical range \\
\cline { 1 - 1 } Low & {$[0,0,3,6]$} \\
Moderate & {$[4,6,8]$} \\
High & {$[6,8,10,10]$}
\end{tabular}

\section{Linguistic variable: Tampering, $T$}

Linguistic value $\quad$ Numerical range

\begin{tabular}{ll}
\hline Low & {$[0,0,2.5,5]$} \\
Moderate & {$[4,6,8]$} \\
High & {$[7,8.5,10,10]$}
\end{tabular}

\section{Linguistic variable: Repudiation, $\mathbf{R}$}

Linguistic value $\quad$ Numerical range

\begin{tabular}{lc}
\hline Low & {$[0,0,2,5]$} \\
Moderate & {$[3.5,6,7.5]$} \\
High & {$[6,8,10,10]$}
\end{tabular}

4. Linguistic variable: Information Disclosure, I

$\begin{array}{lc}\text { Linguistic value } & \text { Numerical range } \\ \text { Low } & {[0,0,2,4.5]} \\ \text { Moderate } & {[3.5,5.5,7.5]} \\ \text { High } & {[6.5,8,10,10]}\end{array}$

5. Linguistic variable: Denial of Service, $D$

Linguistic value $\quad$ Numerical range

Low $\quad[0,0,2.5,5.5]$

Moderate $\quad[4,6,8]$

High $\quad[6.5,9,10,10]$

6. Linguistic variable: Elevation of Privilege, $E$

\begin{tabular}{lc} 
Linguistic value & Numerical range \\
\hline Low & {$[0,0,2.5,6]$} \\
Moderate & {$[4,6,8]$} \\
High & {$[6,8.5,10,10$}
\end{tabular}

7. Linguistic variable: Threat level

Linguistic value Numerical range

Very low $\quad[0,0,30]$

Low $\quad[0,20,40]$

Rather low $\quad[25,35,45]$

Medium [40, 50,70$]$

Rather high $\quad[55,65,75]$

High $\quad[60,80,100]$

Very high $\quad[75,100,100]$ 
b. Rule Evaluation: This is the second step where the fuzzified inputs are applied to the antecedents of the fuzzy rules. Since the fuzzy rule has multiple antecedents, fuzzy operator (AND or $\mathrm{OR}$ ) is used to obtain a single number that represents the result of the antecedent evaluation. We apply the AND fuzzy operation (intersection) to evaluate the conjunction of the rule antecedents. Rules added to this system are about 139 which were derived by mapping the six inputs to one output by using conjunction (AND).

c. Aggregation of the rule outputs: This is the process of unification of the outputs of all rules. In other words, we take the membership functions of all the rules consequents previously scaled and combine them into single fuzzy sets (output). Thus, input of the aggregation process is the list of scaled consequent membership functions, and the output is one fuzzy set for each output variable.

d. Defuzzification: This is the last step in the fuzzy inference process, which is the process of transforming a fuzzy output of a fuzzy inference system into a crisp output. Fuzziness helps to evaluate the rules, but the final output this system has to be a crisp number. The input for the defuzzification process is the aggregate output fuzzy set and the out put is a number. This step was done using Centroid technique because it is most commonly used method of defuzzification.

\section{Implementation and Evaluation}

\section{Implementation}

The design is implemented using MATLAB fuzzy logic toolbox. The interfaces of the implementation are presented below:-

a. FIS Editor (Figure 2): This is the window through which a new FIS type with any particular model can be selected, variable can be added, and input or output variable names can be changed. In this case, the chosen model is Mamdani.

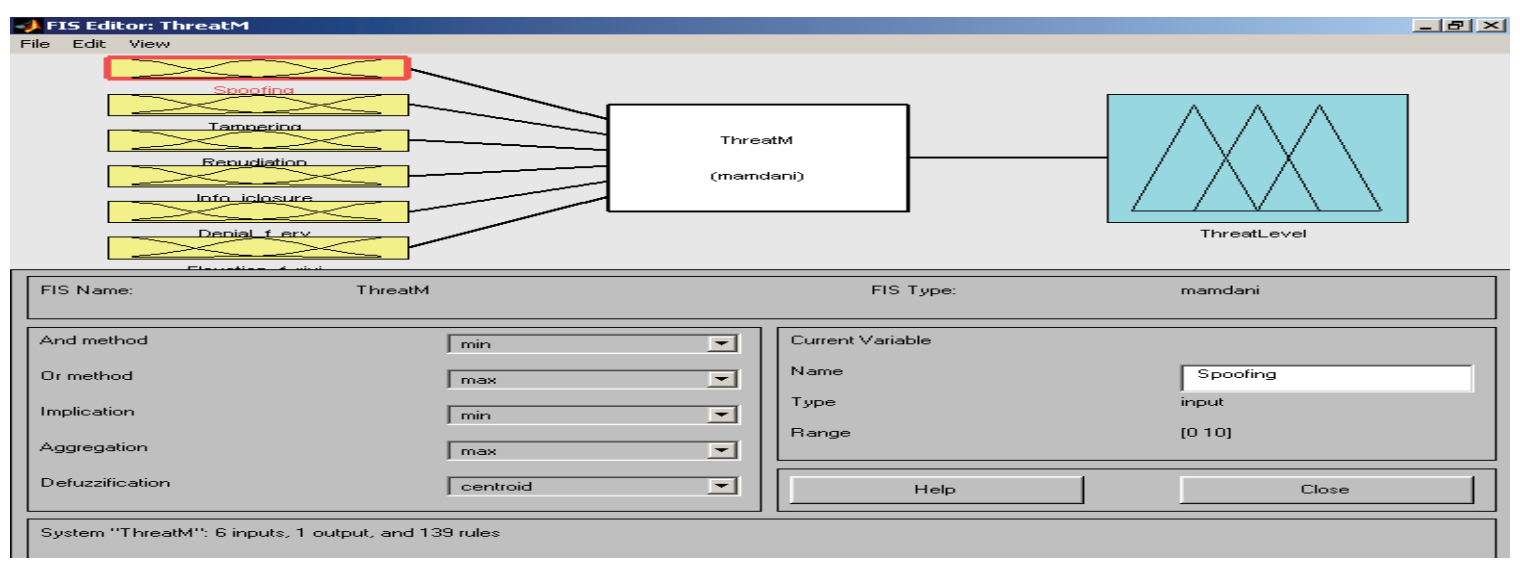

Figure 2: Fuzzy inference Editor

b. Membership function editor (Figures 3 and 4): This is the window through which the input or the output of the membership function can be changed and membership function can be added or removed. It also makes it possible to specify the ranges of each of the variables and membership functions. 


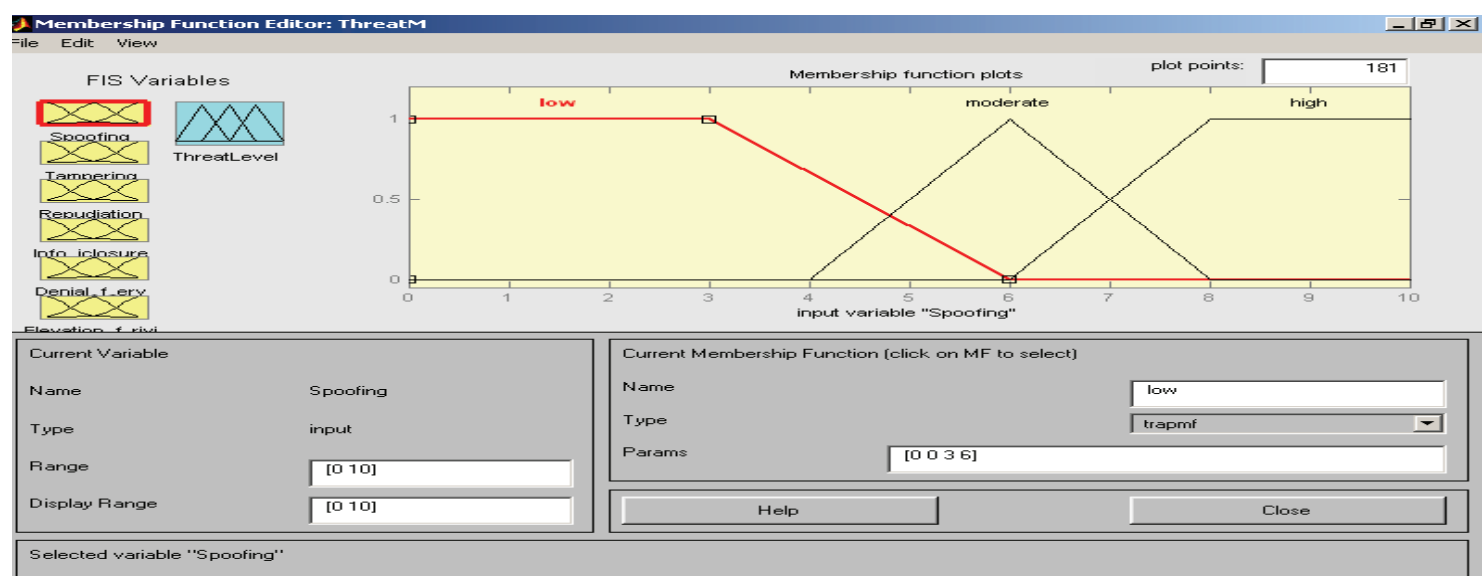

Figure 3: Membership function specification for Spoofing

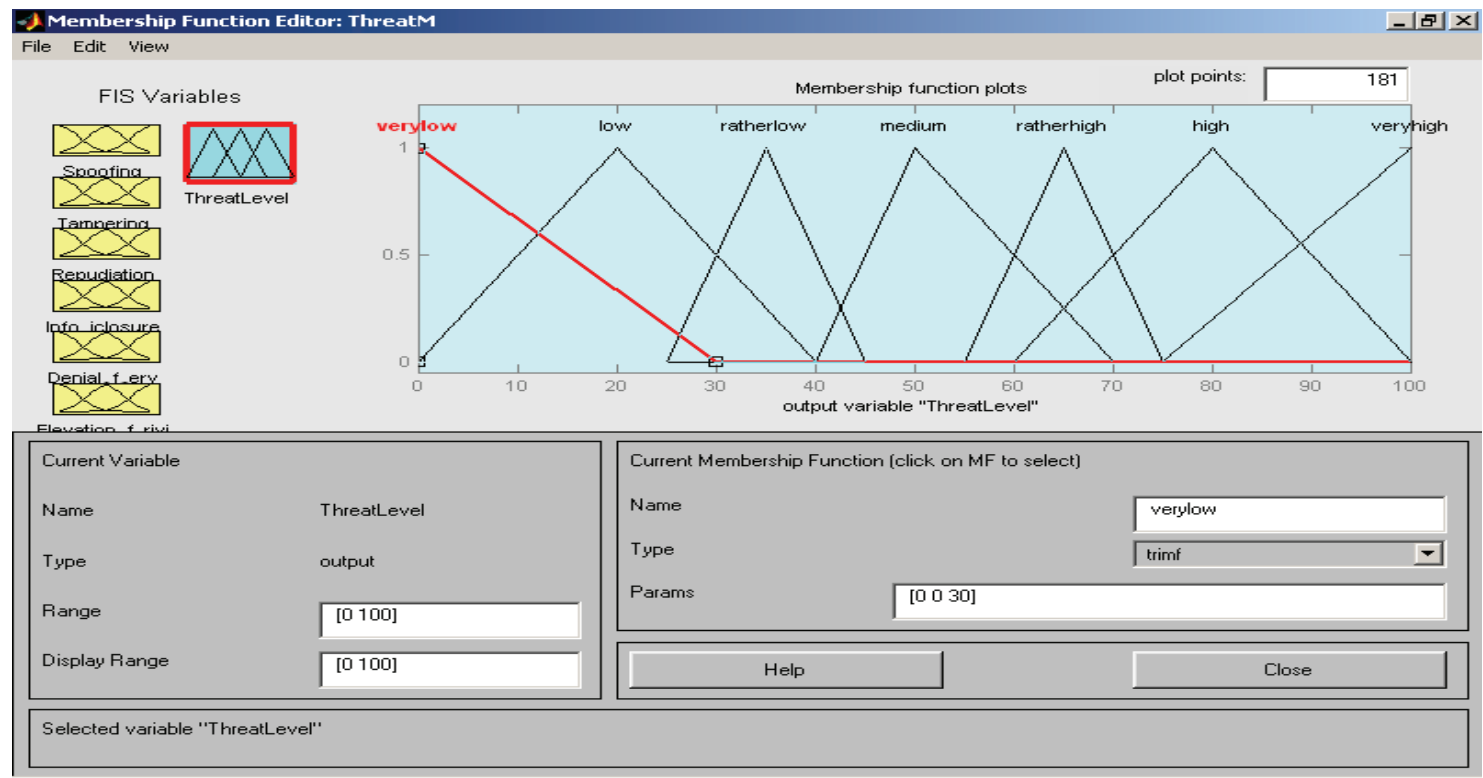

Figure 4: Threat Level (Output) membership function

c. Rule editor (Figure 5): This is used to add, change or delete rules, as the name implies. It provides opportunity to change the connections and weight applied to the rules (the default weight always is 1). 


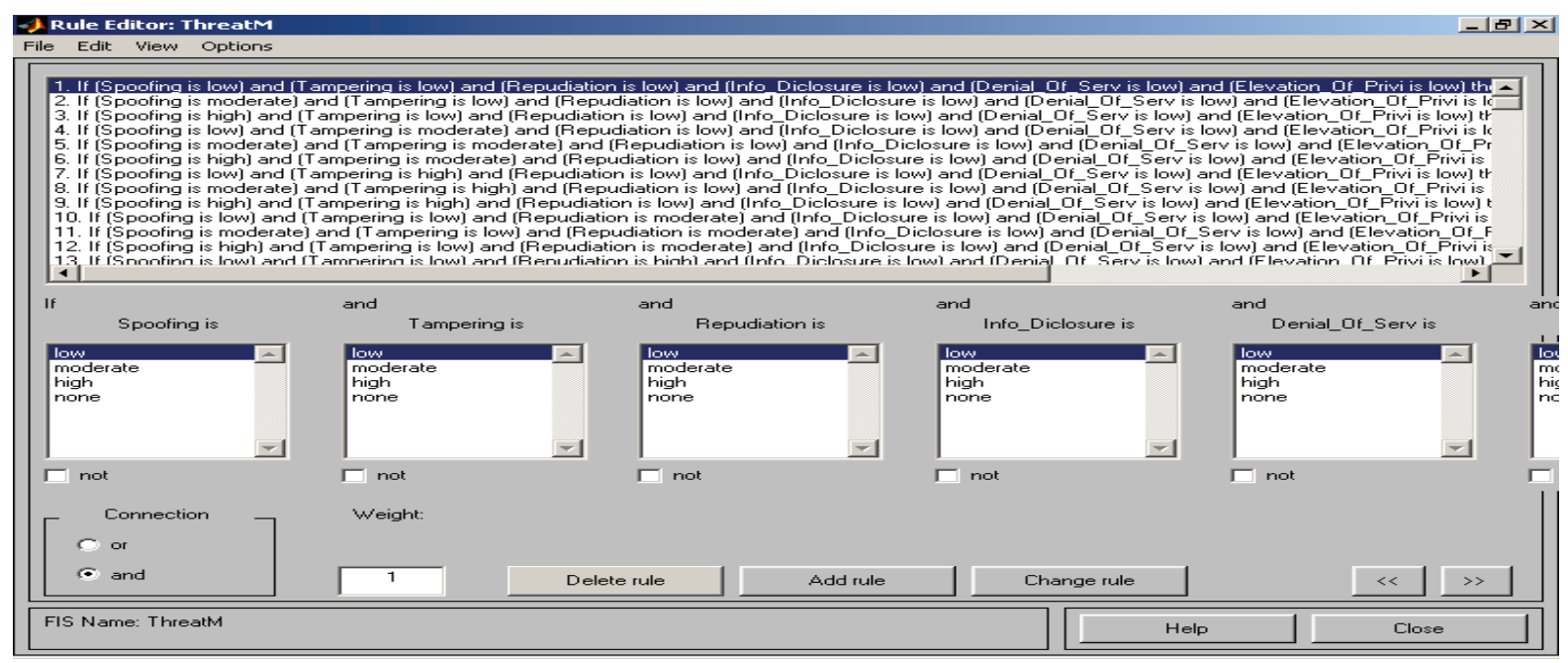

Figure 5: Rules Editor

d. Rule Viewer (Figure 6): The rule viewer shows a graphical representation of each of the variables through all the rules, a representation of the combination of the rules, and a representation of the output from the defuzzification. It also shows the crisp value output of the system. Data are entered for analysis through the Rule Viewer at the Input text field.

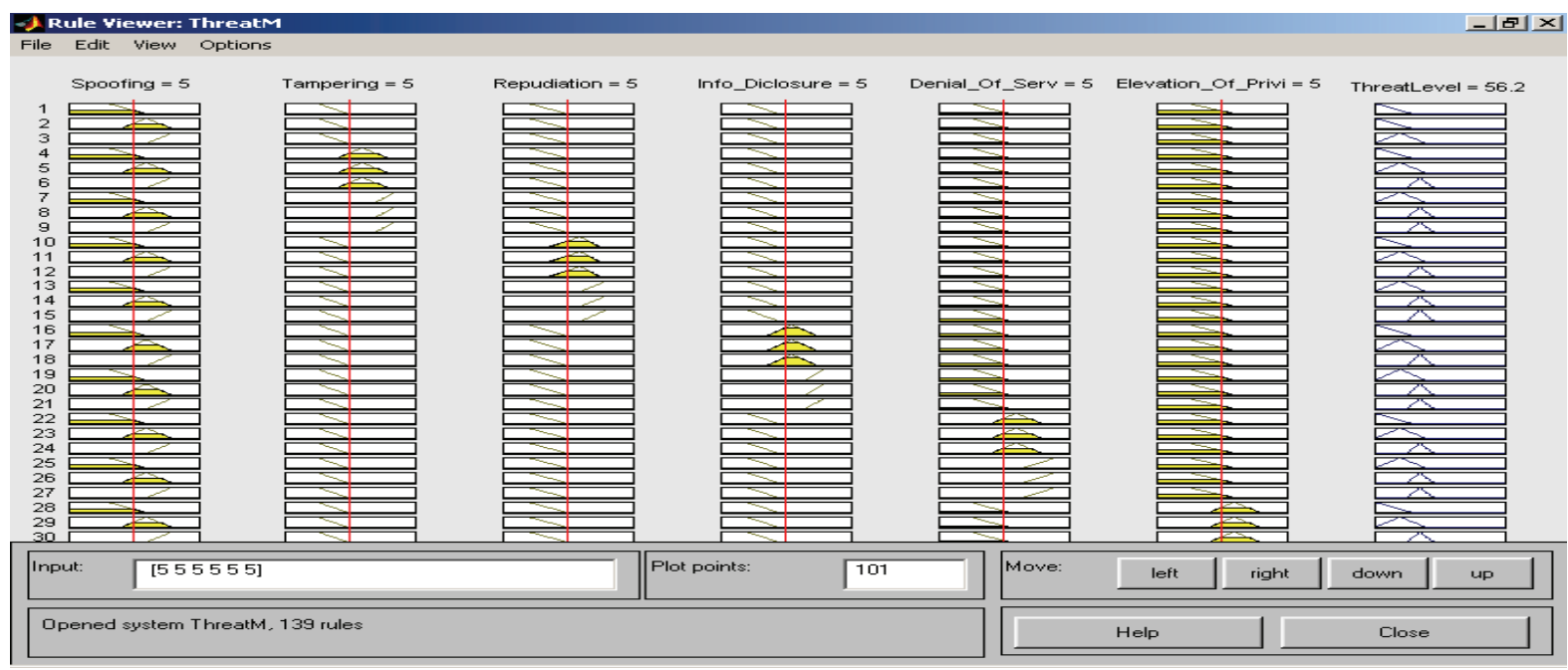

Figure 6: Rule viewer

\section{Evaluation}

The evaluation of the systems shows a system that can be effectively used for qualitative risk assessment. The computer based systems were used to analyze using different assumed values and the output shows that the system is robust enough for the evaluation of the threat level. The output measures the level of an event or activity with the potential to cause harm to their systems. The output is can be a value from 0 to 100 each corresponding to the level of threat.

Five computer systems were analyzed using this system to determine the threat level. Results are shown in Table 1. 
Table 1: Results of the threat level on five computer systems

\begin{tabular}{|l|l|l|}
\hline Systems & Threat level & Implication \\
\hline A & 56.2 & Medium \\
\hline B & 65 & Rather high \\
\hline C & 18.5 & Low \\
\hline D & 90.5 & Very high \\
\hline E & 65 & Rather high \\
\hline
\end{tabular}

\section{Conclusion and Future Work}

\section{Conclusion}

In this work, fuzzy based system was designed to evaluate the threat level of identified threats, because it is impossible to provide assurance for the system and justify security measures incorporated unless the system is analyzed during the designing state of computer based systems. With this system designed, risk analysis has been made easer to perform.

\section{Future Work}

For further research, this system designed can be redesigned using object orientated programming language and other models like DREAD and SWOT model can be used.

\section{References}

Ambler, W. S (2005). Introduction to security threat modeling. Agile Modeling. Available at http://www.agilemodeling.com/artifacts/securityThreatModel.htm

Casteele, S.V. (2005). Threat modeling for web application using STRIDE model.

Davis, N., Howard, M., Humphrey, W., McGraw, G., Redwine Jr., S. T., Zibulski, G., \& Graettinger, C. (2004). Processes to produce secure software: Towards more secure software. A report at National Cyber Security Summit, Vol. 1. Available at http://www.cigital.com/papers/download/secure software process.pdf

De Cock, D., Wouters, K., Schellekens, D., Singelee, D., \& Preneel. B. (2005). Threat modeling for security tokens in web applications. In Proceedings of the IFIP TC6/TC11 International Conference on Communications and Multimedia Security (CMS '04) September 2004, pp 183-193.

Griggs, I. (2004). Browser threat model. Retrieved from http://iang.org/ssl/browser threat model.html

Hoglund, G. \& McGraw, G. (2004). Exploiting software: How to break code. Addison-Wesley Professional.

Klein, S. A. (2005). Position paper on voting system threat modeling. Available at http://vote.nist.gov/threats/papers/threat-modelling.pdf

Sodiya, A.S, Onashoga, S.A, \& Ajayi, O.B (2006). Toward Building Secure Software Products. Journal of issues in Informing Science and Information Technology, 3, 635-646. Available at http://informingscience.org/proceedings/InSITE2006/IISITSodi143.pdf

Swiderski, F. \& Snyder, W. (2004). Threat modeling. Microsoft Press Professional Book Series 


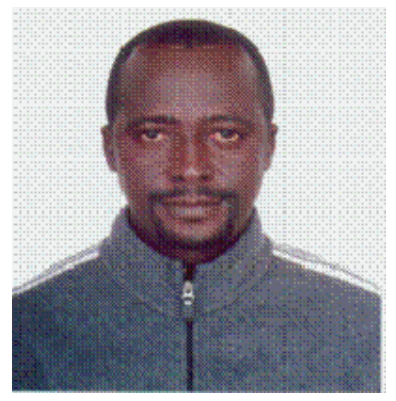

\section{Biographies}

Dr. Adesina Simon Sodiya is presently a lecturer in the department of Computer Science, University of Agriculture, Abeokuta. He has published in both local and international journals. His research areas are Computer Security, Artificial Intelligence, Software Engineering, Data mining.

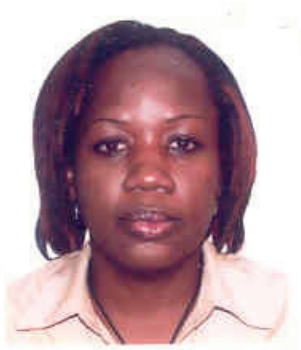

Onashoga, Saidat Adebukola (nee OKUNLAYA, formerly IBRAHIM, S. A.) has worked as a lecturer in the Department of Computer Science, University of Agriculture, Abeokuta, Nigeria for the past six years. She had her degrees in University of Agriculture, Abeokuta, Nigeria with M.Sc. in Computer Science (2004), B.Sc. Mathematical Science (Computer Science option, 2000). She is currently on her Ph.D. programme in Computer Science in the same University. She has published in both International and local journals. Her current research interests include Network Security, Data Mining and Knowledge Management. She is married.

B. A. Oladunjoye [Biography not available] 